\title{
(Anti)de Sitter/Poincaré symmetries and representations from Poincaré/Galilei through a classical deformation approach
}

\author{
Francisco J. Herranz ${ }^{\dagger}$ and Mariano Santander ${ }^{\ddagger}$ \\ † Departamento de Física, Escuela Politécnica Superior \\ Universidad de Burgos, E-09001 Burgos, Spain \\ ¥Departamento de Física Teórica, Facultad de Ciencias \\ Universidad de Valladolid, E-47011 Valladolid, Spain
}

\begin{abstract}
A classical deformation procedure, based on universal enveloping algebras, Casimirs and curvatures of symmetrical homogeneous spaces, is applied to several cases of physical relevance. Starting from the $(3+1) D$ Galilei algebra, we describe at the level of representations the process leading to its two physically meaningful deformed neighbours. The Poincaré algebra is obtained by introducing a negative curvature in the flat Galilean phase space (or space of worldlines), while keeping a flat spacetime. To be precise, starting from a representation of the Galilei algebra with both Casimirs different from zero, we obtain a representation of the Poincaré algebra with both Casimirs necessarily equal to zero. The Poincaré angular momentum, Pauli-Lubanski components, position and velocity operators, etc. are expressed in terms of 'Galilean' operators through some expressions deforming the proper Galilean ones. Similarly, the Newton-Hooke algebras appear by endowing spacetime with a non-zero curvature, while keeping a flat phase space. The same approach, starting from the $(3+1) \mathrm{D}$ Poincaré algebra provides representations of the (anti)de Sitter as Poincaré deformations.
\end{abstract}

KEYWORDS: curvature, spacetime, phase space, representation, Casimir, Lie algebras, Galilei, Poincaré, de Sitter, contraction, deformation

PACS: $\quad 02.20 . \mathrm{Sv} \quad$ 02.20.Qs 11.30.-j 


\section{Introduction}

Classical deformations of Lie algebras can be considered as the opposite process to Lie algebra contractions. In general, starting from a Lie algebra a contraction gives rise to another more abelian algebra by making some structure constants vanish $[1,2,3,4,5$, $6,7]$, while a deformation goes to another less abelian algebra 'producing' some non-zero structure constants $[7,8,9,10,11,12,13,14]$. The idea of contractions of Lie algebras and groups historically appeared in relation with the non-relativistic limit which brings relativistic mechanics (Poincaré group) to classical mechanics (Galilei group) $[1,2,15]$. In the framework of kinematical algebras the scheme of contractions is well known $[16,17]$ : starting from the (anti)de Sitter algebra a sequence of different contractions leads to Poincaré, Newton-Hooke, Galilei, ..., ending up at the last stage in the so called Static algebra. From the viewpoint of the graded contraction theory $[4,5]$, the $(3+1) \mathrm{D}$ case has been studied in [18], and kinematical contractions in arbitrary dimension have been obtained in [19].

The theory of deformations $[8,10,12]$ has been mainly developed in connection with cohomology techniques which relate Lie algebra cohomology groups to Lie algebra deformations [11]. This procedure shows in a systematic way which non-zero Lie brackets can be set different from zero for a given Lie algebra leading to all its possible deformations. By following this approach all the $(3+1)$ D Galilean deformations were obtained in [20]. Recently, classical deformations (within this type of procedures) and contractions have been studied altogether in [21] (see also references therein).

In another approach to deformations, one tries to obtain explicit realizations of a deformed Lie algebra in terms of the universal enveloping algebra of the initial one [7, 9], but as yet these have not been fully systematized and there is no general constructive theory. The procedure given in $[7,9]$ provides the deformations from the universal enveloping algebra of the inhomogeneous pseudo-orthogonal algebra $\mathfrak{i s o}(p, q)$ to the semisimple ones $\mathfrak{s o}(p+1, q)$ (with $p+q=N)$; these contain as particular cases those deformations starting from the Euclidean algebra and leading to either the elliptic or hyperbolic ones as well as the deformations from Poincaré to both de Sitter algebras. A different method [13] enables one to perform the deformations $\mathfrak{t}_{q p}(\mathfrak{s o}(p) \oplus \mathfrak{s o}(q)) \rightarrow \mathfrak{s o}(p, q)$. Similar deformations for unitary algebras can be found in the above references.

In [14] we proposed another deformation method which was formulated in algebraic terms and based, like the above procedures, on the universal enveloping algebra of initial Lie algebras. Nevertheless, the ideas of [14] follow from geometrical considerations which require one to control the deformation by a parameter which has a precise meaning: it is the curvature of some (family of) homogeneous spaces associated with the initial and the deformed Lie algebras. The procedure rests on their (quadratic) Casimir operators. This method was applied to the deformations within the set of $(2+1) \mathrm{D}$ kinematical algebras [16] where the Lie algebras are contractions of $\mathfrak{s o}(4)$. In arbitrary dimension, $\mathfrak{s o}(N+1)$ and its kinematical contractions have as many Casimirs as its rank [22]; only one is quadratic and the others are higher order polynomials in the generators. Then it could happen that a method working for $\mathfrak{s o}(4)$ (the only particular case where the two Casimirs are quadratic) may not be directly extendible to higher dimensions.

In this paper we propose a more general deformation procedure, again based on geometrical ideas (curvatures of homogeneous spaces and Casimirs) which, in principle, can 
be applied to any Lie algebra in any dimension (not only a kinematical one). Such a method, presented in the next section, leads naturally to some operators fulfilling the deformed commutation rules as in $[11,20,21]$, but it also provides explicit expressions of the deformed generators as elements of the universal enveloping algebra of the initial Lie algebra. Thus the resulting expressions may allow a further study of deformations for both operators and representations.

In particular, we apply this procedure to the Galilei algebra in the realistic kinematical dimension, the $(3+1) \mathrm{D}$ case. Hence the basics of the Galilei algebra structure and its main associated symmetric homogeneous spaces (spacetime and phase space) are recalled in section 3. This allows us to produce, in an explicit constructive way, two physically relevant deformations. One reverses the well known non-relativistic limit by obtaining the Poincaré algebra expressed through the universal enveloping Galilei one. Furthermore some Poincaré operators such as Pauli-Lubanski components, angular momentum, position and velocity operators, etc. are expressed in terms of Galilean ones. We remark that these deformed objects are not provided by cohomological procedures as in the Galilean deformations studied in [20]. The other deformation reverses the ordinary zero-curvature limit in the non-relativistic Newton-Hooke spacetimes, whose generators are obtained within the universal enveloping algebra of the centrally extended Galilei algebra. Deformations of representations for both Newton-Hooke algebras are also presented. These two deformations are explicitly developed in sections 4 and 5 , respectively.

Our approach also cover some known cases; for example, the deformations starting from the Poincaré algebra and leading to both (anti)de Sitter algebras [7, 9] and additionally yielding explicitly (anti)de Sitter representations from Poincaré ones. This is described in section 6 . Finally, some remarks and relationships with quantum deformations are pointed out in section 7 .

\section{A classical deformation method}

The Lie algebra deformation method proposed in [14] for the $(2+1) \mathrm{D}$ kinematical algebras $[16,17]$ will be extended here in order to apply to any Lie algebra of arbitrary dimension.

Let $\mathfrak{g}$ be a Lie algebra which is obtained through a contraction from another Lie algebra $\mathfrak{g}^{\prime}, \mathfrak{g}^{\prime} \rightarrow \mathfrak{g}$, with Lie groups $G$ and $G^{\prime}$. Suppose that the contraction corresponds to the vanishing of the curvature $\kappa$ of some non-flat symmetrical homogeneous space $G^{\prime} / H^{\prime}$ and thus leads to a flat space $G / H$, where $H$ and $H^{\prime}$ are the Lie subgroups of the (common) subalgebras invariant under the contraction, and where both homogeneous spaces are of the same rank $r$ (defined by the number of independent invariants of a pair of points in the space under the action of the group [7]; this definition holds for spaces associated to both semisimple and non-semisimple Lie groups; see [23] for the Euclidean space).

Next assume that both $\mathfrak{g}^{\prime}$ and $\mathfrak{g}$ have $m$ functionally independent Casimirs $(m \geq r)$. We shall denote by $\mathcal{C}_{l}(l=1, \ldots, m)$ a linear basis of the center of the universal enveloping algebra of the contracted Lie algebra $\mathfrak{g}$ (with $\kappa=0$ ) and by $\mathcal{C}_{l}^{\prime}$ the corresponding one for the deformed Lie algebra $\mathfrak{g}^{\prime}($ with $\kappa \neq 0)$; the numbering $(l=1, \ldots, m)$ in the set of Casimirs corresponds to increasing order of the $\mathcal{C}_{l}$ as polynomials in the generators, starting from the quadratic one $\mathcal{C}_{1}$ (related to the Killling-Cartan form). Although the 
process involves the choice of a basis, the end result will turn out to be independent of this choice. The deformation process starts from the universal enveloping algebra $U(\mathfrak{g})$ of $\mathfrak{g}$ and looks for a deformed algebra $\mathfrak{g}^{\prime}$ by the following four steps:

(1) Writing the first $r$ deformed Casimirs as polynomials in the curvature $\kappa$ we aim to recover:

$$
\mathcal{C}_{s}^{\prime}=\mathcal{C}_{s}+\kappa \mathcal{J}_{s}^{(1)}+\kappa^{2} \mathcal{J}_{s}^{(2)}+\kappa^{3} \mathcal{J}_{s}^{(3)}+\ldots \quad s=1, \ldots, r
$$

where $\mathcal{C}_{s}, \mathcal{J}_{s}^{(k)}$ are independent of $\kappa$; the $\mathcal{C}_{s}$ are just the contracted Casimirs.

(2) Working in $U(\mathfrak{g})$ within an irreducible representation, consider as a 'deformation seed' some linear combination of the $r$ terms linear in the curvature $\kappa$ :

$$
\mathcal{J}=\sum_{s=1}^{r} \alpha_{s} \mathcal{J}_{s}^{(1)}
$$

where $\alpha_{s}$ are constants to be determined. Notice that any (linear, compatible with the numbering criterion) change in the initial choice of the $\mathcal{C}_{s}^{\prime}$ will lead to the same 'seed' because each of the terms linear in $\kappa$ in the new $\mathcal{C}_{s}^{\prime}$ up to $r$-th are themselves a linear combination of the former; this means that the method is intrinsic, even if it is formulated using a particular basis of the center of the universal enveloping algebra.

(3) For each initial generator $X_{i}$ of $\mathfrak{g}$, define the deformed generators $X_{i}^{\prime}$ of $\mathfrak{g}^{\prime}$ as the elements in $U(\mathfrak{g})$ given by:

$$
X_{i}^{\prime}:=\left\{\begin{array}{lll}
X_{i} & \text { if } & {\left[\mathcal{J}, X_{i}\right]=0} \\
{\left[\mathcal{J}, X_{i}\right]} & \text { if } & {\left[\mathcal{J}, X_{i}\right] \neq 0}
\end{array}\right.
$$

(4) Enforce on the new generators $X_{i}^{\prime}$ the condition that they span a Lie algebra isomorphic to $\mathfrak{g}^{\prime}$. If this is possible at all, this will determine the constants $\alpha_{s}$.

Therefore this four-step procedure firstly lies in the element $\mathcal{J}(2.2)$ which is formed by all the terms 'missing' in $U(\mathfrak{g})$ (according to the rank and the particular homogeneous space chosen for each particular deformation) in order to complete a base of invariants for $U\left(\mathfrak{g}^{\prime}\right)$. And secondly in the expression (2.3) which means that the new (deformed) generators directly come from such a 'missing' part in $U(\mathfrak{g})$, whilst the unchanged ones are those commuting with $\mathcal{J}$ remaining in the same form as in $U(\mathfrak{g})$.

We stress that the resulting expressions (2.3) may allow one to obtain operators and realizations of the deformed algebra $\mathfrak{g}^{\prime}$ in terms of those of $U(\mathfrak{g})$ providing deformations for Lie algebra representations of the initial algebra.

It is worth noticing a main difference of this approach with other proposals $[7,9,13]$ : here not only the second-order $\mathcal{C}_{1}$ but all the Casimir invariants play a role in the deformation process, and the end result is intrinsic and would not change if a different choice for the Casimirs was used. In particular, we shall show that the fourth-order Pauli-Lubanski invariant is essential in order to recover the Poincaré algebra and some representations from the Galilei case. The need of using the higher order Casimirs might explain why the inverse process to the Inönü-Wigner non-relativistic contraction has remained, as a matter of fact, as an unsolved problem in the framework of operators/representations. 
In this respect we recall that it was already clear in the 70's that higher-order Casimirs (and not only the quadratic one) would be involved in higher-rank deformations. This is explicitly stated by Gilmore [7], but apparently this line was not pursued enough.

\section{The Galilei algebra and associated homogeneous spaces}

In order to apply the above method to the $(3+1) \mathrm{D}$ Galilei algebra, $\mathcal{G} \equiv \mathfrak{i i s o}(3)$, we first recall some basics. Let $H, P_{i}, K_{i}$ and $J_{i}$ be the usual generators of Galilean time translation, space translations, boosts and spatial rotations, respectively, with Lie brackets given by

$$
\begin{array}{lll}
{\left[J_{i}, J_{j}\right]=\varepsilon_{i j k} J_{k}} & {\left[J_{i}, P_{j}\right]=\varepsilon_{i j k} P_{k}} & {\left[J_{i}, K_{j}\right]=\varepsilon_{i j k} K_{k}} \\
{\left[P_{i}, P_{j}\right]=0} & {\left[P_{i}, K_{j}\right]=0} & {\left[K_{i}, K_{j}\right]=0} \\
{\left[H, P_{i}\right]=0} & {\left[H, K_{i}\right]=-P_{i}} & {\left[H, J_{i}\right]=0}
\end{array}
$$

where $i, j, k=1,2,3$; sums over repeated indices will be generally assumed from now on. Hereafter we consider the $3+1$ notation and any 3-'vector' is denoted as $\mathbf{X}=\left(X_{1}, X_{2}, X_{3}\right)$; its square and its scalar and cross products with other vector, say $\mathbf{Y}=\left(Y_{1}, Y_{2}, Y_{3}\right)$, are

$$
\begin{aligned}
& \mathbf{X}^{2}=X_{1}^{2}+X_{2}^{2}+X_{3}^{2} \quad|\mathbf{X}|=\left(X_{1}^{2}+X_{2}^{2}+X_{3}^{2}\right)^{1 / 2} \\
& \mathbf{X} \cdot \mathbf{Y}=X_{1} Y_{1}+X_{2} Y_{2}+X_{3} Y_{3} \quad \mathbf{X} \wedge \mathbf{Y}=\mathbf{Z} \quad Z_{i}=\varepsilon_{i j k} X_{j} Y_{k} .
\end{aligned}
$$

Note that $\mathbf{X}$ and $\mathbf{Y}$ are generically non-commutative objects so that the ordering in these products must be preserved.

The Galilei algebra $\mathcal{G}$ has two Casimir invariants (quadratic and quartic) which read:

$$
\mathcal{C}_{1}=\mathbf{P}^{2} \quad \mathcal{C}_{2}=\mathbf{W}^{2} \quad \mathbf{W}=\mathbf{K} \wedge \mathbf{P}
$$

corresponding to the square of the linear momentum $\mathbf{P}$ (non-relativistic kinetic energy) and to the square of the angular momentum $\mathbf{W}$, respectively.

Further computations within the universal enveloping Galilei algebra require use of the identities:

$$
\mathbf{P} \cdot \mathbf{W}=0 \quad \mathbf{K} \cdot \mathbf{W}=0
$$

which imply that

$$
|\mathbf{P} \wedge \mathbf{W}|=|\mathbf{P}||\mathbf{W}| \quad|\mathbf{K} \wedge \mathbf{W}|=|\mathbf{K}||\mathbf{W}| .
$$

We shall also need the following Lie brackets between the Galilei generators, the angular momentum components $W_{i}$ and the products $\mathbf{J} \cdot \mathbf{P}$ and $\mathbf{J} \cdot \mathbf{W}$ :

$$
\begin{gathered}
{\left[W_{i}, H\right]=\left[W_{i}, P_{j}\right]=\left[W_{i}, K_{j}\right]=\left[W_{i}, W_{j}\right]=0 \quad\left[W_{i}, J_{j}\right]=\varepsilon_{i j k} W_{k}} \\
{[\mathbf{J} \cdot \mathbf{P}, H]=\left[\mathbf{J} \cdot \mathbf{P}, P_{i}\right]=\left[\mathbf{J} \cdot \mathbf{P}, J_{i}\right]=0 \quad\left[\mathbf{J} \cdot \mathbf{P}, K_{i}\right]=W_{i}} \\
{\left[\mathbf{J} \cdot \mathbf{P}, W_{i}\right]=-\varepsilon_{i j k} P_{j} W_{k}} \\
{[\mathbf{J} \cdot \mathbf{W}, H]=\left[\mathbf{J} \cdot \mathbf{W}, J_{i}\right]=\left[\mathbf{J} \cdot \mathbf{W}, W_{i}\right]=0} \\
{\left[\mathbf{J}, P_{i}\right]=\varepsilon_{i j k} P_{j} W_{k} \quad\left[\mathbf{J} \cdot \mathbf{W}, K_{i}\right]=\varepsilon_{i j k} K_{j} W_{k} \quad[\mathbf{J} \cdot \mathbf{W}, \mathbf{J} \cdot \mathbf{P}]=\varepsilon_{i j k} J_{i} P_{j} W_{k} .}
\end{gathered}
$$

Other commutators which involve the Casimir invariants are given by:

$$
\begin{array}{ll}
{\left[\mathbf{J} \cdot \mathbf{P}, \varepsilon_{i j k} P_{j} W_{k}\right]=\mathcal{C}_{1} W_{i}} & {\left[\mathbf{J} \cdot \mathbf{P}, \varepsilon_{i j k} K_{j} W_{k}\right]=\mathbf{K} \cdot \mathbf{P} W_{i}} \\
{\left[\mathbf{J} \cdot \mathbf{W}, \varepsilon_{i j k} P_{j} W_{k}\right]=-\mathcal{C}_{2} P_{i}} & {\left[\mathbf{J} \cdot \mathbf{W}, \varepsilon_{i j k} K_{j} W_{k}\right]=-\mathcal{C}_{2} K_{i} .}
\end{array}
$$


The involutive automorphisms parity $\mathrm{P}$ and the product $\mathrm{PT}[16]$ (where $\mathrm{T}$ is the timereversal)

$$
\begin{array}{ll}
\text { PT : } & (H, \mathbf{P}, \mathbf{K}, \mathbf{J}) \rightarrow(-H,-\mathbf{P}, \mathbf{K}, \mathbf{J}) \\
\text { P : } & (H, \mathbf{P}, \mathbf{K}, \mathbf{J}) \rightarrow(H,-\mathbf{P},-\mathbf{K}, \mathbf{J})
\end{array}
$$

determine two Cartan decompositions of the Galilei Lie algebra $\mathcal{G}$

$$
\begin{array}{llll}
\text { PT : } & \mathcal{G}=\mathfrak{p}^{(1)} \oplus \mathfrak{h}^{(1)} & \mathfrak{p}^{(1)}=\langle H, \mathbf{P}\rangle & \mathfrak{h}^{(1)}=\langle\mathbf{K}, \mathbf{J}\rangle \\
\text { P : } & \mathcal{G}=\mathfrak{p}^{(2)} \oplus \mathfrak{h}^{(2)} & \mathfrak{p}^{(2)}=\langle\mathbf{P}, \mathbf{K}\rangle & \mathfrak{h}^{(2)}=\langle H, \mathbf{J}\rangle=\langle H\rangle \oplus\langle\mathbf{J}\rangle
\end{array}
$$

fulfilling

$$
\left[\mathfrak{h}^{(l)}, \mathfrak{h}^{(l)}\right] \subset \mathfrak{h}^{(l)} \quad\left[\mathfrak{h}^{(l)}, \mathfrak{p}^{(l)}\right] \subset \mathfrak{p}^{(l)} \quad\left[\mathfrak{p}^{(l)}, \mathfrak{p}^{(l)}\right]=0 \quad l=1,2 .
$$

Consequently, the Galilei group $G \equiv I I S O(3)$ is the motion group of the two following symmetrical homogeneous spaces with dimension $D_{l}$, curvature $\kappa_{l}$ and rank $r_{l}$ :

$$
\begin{array}{lllll}
\mathcal{S}^{(1)}=G / H^{(1)}=I I S O(3) / I S O(3) & D_{1}=4 & \kappa_{1}=0 & r_{1}=1 \\
\mathcal{S}^{(2)}=G / H^{(2)}=I I S O(3) /(\mathbb{R} \otimes S O(3)) & D_{2}=6 & \kappa_{2}=0 & r_{2}=2 .
\end{array}
$$

The Galilei subgroups $H^{(1)}, H^{(2)}$ (whose Lie algebras are $\mathfrak{h}^{(1)}, \mathfrak{h}^{(2)}$ ) are the stabilizer subgroups of an event and of a time-like line, respectively. Therefore the flat rank-one space $\mathcal{S}^{(1)}$ is identified with the $(3+1) \mathrm{D}$ Galilean spacetime, while the flat rank-two space $\mathcal{S}^{(2)}$ is the $6 \mathrm{D}$ space of time-like lines in the Galilean spacetime $\mathcal{S}^{(1)}$; we remark that $\mathcal{S}^{(2)}$ itself is the (classical) phase space of a free Galilean particle. Hence this geometric approach makes two types of spaces to appear: spacetime, on one side, and phase spaces on the other. For a description of the structure of phase spaces behind the possible homogeneous spacetimes (Galilei, Newton-Hooke, Minkowski, (anti)de Sitter) as ranktwo spaces, see [24]. The phase spaces are the basic objects in the geometric quantization program, and through identification to orbits of the coadjoint action an interesting link to the study and classification of representations, the 'orbit method', has been developed by Souriau, Kostant and Kirillov (for a recent overview of this method, see [25]). Our aim here is more specific as we are not dealing with quantization at all.

Hence, as it is well known, two possible deformations arise from Galilei in a natural way. First, if we consider the Galilean spacetime $\mathcal{S}^{(1)}$ we can try to reach a kinematics whose associated spacetime has a non-zero curvature but keeps a flat phase space $\mathcal{S}^{(2)}$; that is, we keep non-relativistic (classical) mechanics. The resulting symmetries span the two Newton-Hooke (NH) algebras which are obtained through rank-one non-relativistic deformations that provide curved spacetimes with curvature $\kappa_{1}= \pm 1 / \tau^{2}$ (where $\tau$ is the universe 'radius') but are still with absolute time. The opposite process is the spacetime contraction $\tau \rightarrow \infty$ in the NH algebras [16].

The second possibility is to start with the rank-two space $\mathcal{S}^{(2)}$ and introducing a (negative) curvature in $\mathcal{S}^{(2)}$ as $\kappa_{2}=-1 / c^{2}$, while keeping a flat spacetime $\mathcal{S}^{(1)}$, and so reaching the Poincaré algebra. Hence the usual (flat) phase space supporting classical mechanics becomes the proper 'curved' phase space underlying relativistic mechanics [24]. Alternatively, this process can be seen as a relativistic deformation which gives rise to the Minkowskian spacetime, still a flat universe (spacetime) but now without absolute time. This is exactly the opposite process to the well known non-relativistic limit or speed-space contraction $c \rightarrow \infty[1,2,3,16]$.

In the next two sections we discuss in full explicit detail both types of Lie algebra deformations together with a study of the corresponding deformation of representations. 


\section{Poincaré from Galilei}

In this section we will see how the proposed deformation procedure leads to representations of the Poincaré algebra starting from those representations of the Galilei algebra with non-zero values for both Casimirs. These Galilei representations will be termed here as 'ordinary' just to emphasize that they are in some sense the 'generic ones'. At the representation level the procedure requires to restrict oneself to those 'ordinary' representations of the Galilei algebra to start with, and leads precisely to 'non-ordinary' representations of the Poincaré algebra, with both Casimirs vanishing.

The Lie brackets of the $(3+1)$ D Poincaré algebra $\mathcal{P} \equiv \mathfrak{i s o}(3,1)$ read

$$
\begin{aligned}
& {\left[J_{i}^{\prime}, J_{j}^{\prime}\right]=\varepsilon_{i j k} J_{k}^{\prime} \quad\left[J_{i}^{\prime}, P_{j}^{\prime}\right]=\varepsilon_{i j k} P_{k}^{\prime} \quad\left[J_{i}^{\prime}, K_{j}^{\prime}\right]=\varepsilon_{i j k} K_{k}^{\prime}} \\
& {\left[P_{i}^{\prime}, P_{j}^{\prime}\right]=0} \\
& {\left[P_{i}^{\prime}, K_{j}^{\prime}\right]=-\frac{1}{c^{2}} \delta_{i j} H^{\prime}} \\
& {\left[K_{i}^{\prime}, K_{j}^{\prime}\right]=-\frac{1}{c^{2}} \varepsilon_{i j k} J_{k}^{\prime}} \\
& {\left[H^{\prime}, P_{i}^{\prime}\right]=0} \\
& {\left[H^{\prime}, K_{i}^{\prime}\right]=-P_{i}^{\prime}}
\end{aligned}
$$

The two Poincaré Casimir invariants are given by

$$
\begin{aligned}
& \mathcal{C}_{1}^{\prime}=\mathbf{P}^{\prime 2}-\frac{1}{c^{2}} H^{\prime 2} \quad \mathcal{C}_{2}^{\prime}=\mathbf{W}^{\prime 2}-\frac{1}{c^{2}} W_{0}^{\prime 2} \\
& W_{0}^{\prime}=\mathbf{J}^{\prime} \cdot \mathbf{P}^{\prime} \quad W_{i}^{\prime}=-\frac{1}{c^{2}} H^{\prime} J_{i}^{\prime}+\varepsilon_{i j k} K_{j}^{\prime} P_{k}^{\prime} .
\end{aligned}
$$

For an elementary system, the Casimir $\mathcal{C}_{1}^{\prime}$ is, up to a factor, the square of the energymomentum 4-vector $\left(H^{\prime}, \mathbf{P}^{\prime}\right)$; with the conventions here, this Casimir is equal to minus the system rest mass squared, while $\mathcal{C}_{2}^{\prime}$ comes similarly from the square of the Pauli-Lubanski 4 -vector $\left(W_{0}^{\prime}, \mathbf{W}^{\prime}\right)$, and is the square of the angular momentum in the rest frame; both quantities are the Poincaré invariants characterizing elementary systems.

The Cartan decompositions (3.10) also hold for the Poincaré algebra, though in this case the vector subspaces $\mathfrak{p}^{(l)}$ satisfy $\left[\mathfrak{p}^{(1)}, \mathfrak{p}^{(1)}\right]=0$ and $\left[\mathfrak{p}^{(2)}, \mathfrak{p}^{(2)}\right] \subset \mathfrak{h}^{(2)}$ in the relations (3.11). Therefore the Poincaré group $P \equiv I S O(3,1)$ is the motion group of the symmetric homogeneous spaces defined by

$$
\begin{array}{llll}
\mathcal{S}^{(1)}=P / H^{(1)}=I S O(3,1) / S O(3,1) & D_{1}=4 & \kappa_{1}=0 & r_{1}=1 \\
\mathcal{S}^{(2)}=P / H^{(2)}=I S O(3,1) /(\mathbb{R} \otimes S O(3)) & D_{2}=6 & \kappa_{2}=-1 / c^{2} & r_{2}=2
\end{array}
$$

which are identified with the $(3+1) \mathrm{D}$ Minkowskian spacetime and the $6 \mathrm{D}$ (relativistic) phase space (or space of time-like lines), respectively.

The non-relativistic limit $c \rightarrow \infty$ leads to the contraction $\mathcal{P} \rightarrow \mathcal{G}$ and makes equal to zero the curvature of $\mathcal{S}^{(2)}$, so that the commutators $\left[P_{i}^{\prime}, K_{j}^{\prime}\right],\left[K_{i}^{\prime}, K_{j}^{\prime}\right]$ are equal to zero in $\mathcal{G}$. Our aim now is to reverse this process, that is, to try to recover $\mathcal{P}$ inside the universal enveloping algebra of $\mathcal{G}, U(\mathcal{G})$, thus introducing the relativistic constant $c$.

\subsection{The relativistic Galilei deformation}

As the intended deformation $U(\mathcal{G}) \rightarrow \mathcal{P}$, which would produce the Poincaré algebra from the Galilei one, is a rank-two deformation, the first step of the deformation method (2.1) requires us to rewrite both Poincaré Casimirs so as to explicitly display the powers of the 
curvature $-1 / c^{2}$ of the phase space $\mathcal{S}^{(2)}$; this gives the $\mathcal{J}_{i}^{(l)}$ :

$$
\begin{aligned}
& \mathcal{C}_{1}^{\prime}=\mathcal{C}_{1}-\frac{1}{c^{2}} \mathcal{J}_{1}^{(1)} \quad \mathcal{J}_{1}^{(1)}=H^{2} \\
& \mathcal{C}_{2}^{\prime}=\mathcal{C}_{2}-\frac{1}{c^{2}} \mathcal{J}_{2}^{(1)}+\left(-\frac{1}{c^{2}}\right)^{2} \mathcal{J}_{2}^{(2)} \quad \mathcal{J}_{2}^{(1)}=2 H \mathbf{J} \cdot \mathbf{W}+(\mathbf{J} \cdot \mathbf{P})^{2} \quad \mathcal{J}_{2}^{(2)}=H^{2} \mathbf{J}^{2}
\end{aligned}
$$

where $\mathcal{C}_{1}, \mathcal{C}_{2}$ are the Galilei Casimirs (3.3). We now consider the linear combination (2.2)

$$
\mathcal{J}=\alpha_{1} \mathcal{J}_{1}^{(1)}+\alpha_{2} \mathcal{J}_{2}^{(1)}=\alpha_{1} H^{2}+\alpha_{2}\left(2 H \mathbf{J} \cdot \mathbf{W}+(\mathbf{J} \cdot \mathbf{P})^{2}\right)
$$

to be used as a seed to provide generators $X_{k}^{\prime}$ intended to close a Poincaré algebra. These are the elements of $U(\mathcal{G})$ obtained by following the third step (2.3), which requires commuting $\mathcal{J}$ with the Galilei generators $X_{k}$ :

$$
\begin{aligned}
& H^{\prime}=H \quad J_{i}^{\prime}=J_{i} \quad P_{i}^{\prime}=2 \alpha_{2} H \varepsilon_{i j k} P_{j} W_{k} \\
& K_{i}^{\prime}=-2 \alpha_{1} H P_{i}+2 \alpha_{2}\left(\mathbf{J} \cdot \mathbf{P} W_{i}-\mathbf{J} \cdot \mathbf{W} P_{i}+H \varepsilon_{i j k} K_{j} W_{k}+\frac{3}{2} \varepsilon_{i j k} P_{j} W_{k}\right) .
\end{aligned}
$$

The last step is to enforce the deformed generators (4.5) to fulfil the Lie brackets of the Poincaré algebra (4.1); the final result is established by:

Proposition 1. For a given irreducible representation of the Galilei algebra $\mathcal{G}$ with nonvanishing Casimirs $\mathcal{C}_{1} \neq 0, \mathcal{C}_{2} \neq 0$, the generators defined by $(4.5)$ close the $(3+1) D$ Poincaré algebra $\mathcal{P}$ whenever the constants $\alpha_{1}, \alpha_{2}$ are related to the Casimirs values by

$$
\alpha_{1} \mathcal{C}_{1}+\alpha_{2} \mathcal{C}_{2}=0 \quad \alpha_{2}^{2}=\frac{1}{4 c^{2} \mathcal{C}_{1} \mathcal{C}_{2}}
$$

Notice that this process will only work for those irreducible representations of the starting Galilei algebra with both Casimirs different from zero. There is no a standard naming for these representations, but as this condition is essential in our approach, we will refer here to these as 'ordinary' Galilei representations.

The proof of this result is a direct computation. Some generators stay unchanged in this deformation and span the subalgebra $\mathfrak{h}^{(2)}=\langle H, \mathbf{J}\rangle$. By taking into account the second Cartan decomposition (3.10) it can be checked that the assumptions of the proposition 1 of [14] are automatically fulfilled, which implies that the Lie brackets $\left[\mathfrak{h}^{(2)}, \mathfrak{h}^{(2)}\right]$ and $\left[\mathfrak{h}^{(2)}, \mathfrak{p}^{(2)}\right]$ remain in the same form as in the Galilei algebra, as it should be for Poincaré. Therefore, we have only to compute the commutators involving generators in $\mathfrak{p}^{(2)}:\left[P_{i}^{\prime}, P_{j}^{\prime}\right]$, $\left[P_{i}^{\prime}, K_{j}^{\prime}\right]$ and $\left[K_{i}^{\prime}, K_{j}^{\prime}\right]$.

As it is shown in (3.6), H, $P_{i}$ and $W_{j}$ commute amongst themselves, so that $\left[P_{i}^{\prime}, P_{j}^{\prime}\right]=$ 0 . Let us compute $\left[P_{i}^{\prime}, K_{l}^{\prime}\right]$; by using (3.6) and (3.7) we obtain that

$$
\begin{aligned}
{\left[P_{i}^{\prime}, K_{l}^{\prime}\right]=} & -4 \alpha_{2}^{2} \varepsilon_{i j k} H\left[P_{j}, \mathbf{J} \cdot \mathbf{W}\right] P_{l} W_{k}+4 \alpha_{2}^{2} \varepsilon_{i j k} H P_{j}\left[W_{k}, \mathbf{J} \cdot \mathbf{P}\right] W_{l} \\
& \quad+4 \alpha_{2}^{2} \varepsilon_{i j k} \varepsilon_{l m n} H\left[H, K_{m}\right] P_{j} W_{k} W_{n} \\
= & 4 \alpha_{2}^{2} H \varepsilon_{i j k}\left(\varepsilon_{j m n} P_{l} W_{k}+\varepsilon_{k m n} P_{j} W_{l}-\varepsilon_{l m n} P_{j} W_{k}\right) P_{m} W_{n} .
\end{aligned}
$$

Thus, if $i \neq l,\left[P_{i}^{\prime}, K_{l}^{\prime}\right]=0$, but whenever $i=l$ the Galilei Casimirs (3.3) can be recovered in the commutator as

$$
\left[P_{i}^{\prime}, K_{i}^{\prime}\right]=-4 \alpha_{2}^{2} H \mathbf{P}^{2} \mathbf{W}^{2}=-4 \alpha_{2}^{2} H \mathcal{C}_{1} \mathcal{C}_{2}=-4 \alpha_{2}^{2} \mathcal{C}_{1} \mathcal{C}_{2} H^{\prime}
$$


and this will coincide with the expected Poincaré commutation relation whenever

$$
-4 \alpha_{2}^{2} \mathcal{C}_{1} \mathcal{C}_{2}=-\frac{1}{c^{2}}
$$

which leads to the second relation of (4.6). Next, let us compute

$$
\begin{aligned}
{\left[K_{i}^{\prime}, K_{l}^{\prime}\right]=} & 8 \alpha_{1} \alpha_{2} H\left(\varepsilon_{l j k} P_{i}-\varepsilon_{i j k} P_{l}\right) P_{j} W_{k}-4 \alpha_{2}^{2} H \mathcal{C}_{2} \varepsilon_{i l k} W_{k} \\
& -4 \alpha_{2}^{2} H \varepsilon_{i j k} \varepsilon_{l m n} \varepsilon_{s j m} W_{n} W_{s} W_{k}-4 \alpha_{2}^{2}[\mathbf{J} \cdot \mathbf{W}, \mathbf{J} \cdot \mathbf{P}]\left(P_{i} W_{l}-P_{l} W_{i}\right) \\
& +4 \alpha_{2}^{2}\left\{\mathbf{J} \cdot \mathbf{W}\left(\varepsilon_{l j k} P_{i}-\varepsilon_{i j k} P_{l}\right) P_{j} W_{k}+\mathbf{J} \cdot \mathbf{P}\left(\varepsilon_{l j k} W_{i}-\varepsilon_{i j k} W_{l}\right) W_{j} P_{k}\right\} .
\end{aligned}
$$

By using identities (3.4), simplifying, grouping terms in order to construct the Galilei Casimirs and using the recently obtained relation we find that

$$
\begin{aligned}
{\left[K_{i}^{\prime}, K_{l}^{\prime}\right]=} & -8 \alpha_{2} H \varepsilon_{i l k}\left(\alpha_{1} \mathcal{C}_{1}+\alpha_{2} \mathcal{C}_{2}\right) W_{k}-4 \alpha_{2}^{2} \varepsilon_{i l k}\left(\mathbf{J} \cdot \mathbf{W} \mathcal{C}_{1} W_{k}+\mathbf{J} \cdot \mathbf{P} \mathcal{C}_{2} P_{k}\right) \\
& -4 \alpha_{2}^{2} \varepsilon_{m n s} J_{m} P_{n} W_{s}\left(P_{i} W_{l}-P_{l} W_{i}\right) \\
= & -8 \alpha_{2} H \varepsilon_{i l k}\left\{\alpha_{1} \mathcal{C}_{1}+\alpha_{2} \mathcal{C}_{2}\right\} W_{k}-4 \alpha_{2}^{2} \varepsilon_{i l k} J_{k} \mathcal{C}_{1} \mathcal{C}_{2} \\
= & -8 \alpha_{2} H \varepsilon_{i l k}\left\{\alpha_{1} \mathcal{C}_{1}+\alpha_{2} \mathcal{C}_{2}\right\} W_{k}-\frac{1}{c^{2}} \varepsilon_{i l k} J_{k}^{\prime}
\end{aligned}
$$

so this coincides with the Poincaré commutator whenever

$$
\alpha_{1} \mathcal{C}_{1}+\alpha_{2} \mathcal{C}_{2}=0
$$

Hence proposition 1 is proven.

Consequently, this Galilean deformation reverses the non-relativistic contraction of the Poincaré algebra by introducing a negative curvature $\kappa_{2}=-1 / c^{2}$ in the $6 \mathrm{D}$ Galilean phase space $\mathcal{S}^{(2)}$. Recall that the space $\mathcal{S}^{(2)}$ is rank-two, so its geometry is very different from a rank-one space, either curved or flat [24]. At the level of the Cartan decompositions the deformation gives $\left[\mathfrak{p}^{(2)}, \mathfrak{p}^{(2)}\right]=0 \rightarrow\left[\mathfrak{p}^{(2)}, \mathfrak{p}^{(2)}\right] \subset \mathfrak{h}^{(2)}$.

\subsection{Relativistic Poincaré operators through non-relativistic Galilei ones}

Once the constants $\alpha_{1}$ and $\alpha_{2}$ have been determined, the expressions of the Poincaré generators in terms of those of the universal envolvent algebra $U(\mathcal{G})(4.5)$ of the Galilei algebra can be recast very compactly in vector form. We restrict from the beginning to 'ordinary' representations of the Galilei algebra, with $\mathcal{C}_{1} \neq 0$ and $\mathcal{C}_{2} \neq 0$. Choose the positive root determination for $\alpha_{2}$, and use the notation (3.2), writing $\mathcal{C}_{1}^{1 / 2}=|\mathbf{P}|$ and $\mathcal{C}_{2}^{1 / 2}=|\mathbf{W}|$. For the deformed generators we obtain:

$$
\begin{aligned}
c \mathbf{P}^{\prime} & =H \frac{\mathbf{P} \wedge \mathbf{W}}{|\mathbf{P} \| \mathbf{W}|} \quad H^{\prime}=H \quad \mathbf{J}^{\prime}=\mathbf{J} \\
c \mathbf{K}^{\prime} & =H\left(\frac{|\mathbf{W}|}{\mathbf{P}^{2}} \frac{\mathbf{P}}{|\mathbf{P}|}+\frac{\mathbf{K} \wedge \mathbf{W}}{|\mathbf{P}||\mathbf{W}|}\right)+\left(\frac{\mathbf{J} \cdot \mathbf{P}}{|\mathbf{P}|} \frac{\mathbf{W}}{|\mathbf{W}|}-\frac{\mathbf{J} \cdot \mathbf{W}}{|\mathbf{W}|} \frac{\mathbf{P}}{|\mathbf{P}|}\right)+\frac{3}{2} \frac{\mathbf{P} \wedge \mathbf{W}}{|\mathbf{P}||\mathbf{W}|} \\
& =\frac{1}{|\mathbf{P} \| \mathbf{W}|}\left\{H\left(\frac{\mathbf{W}^{2}}{\mathbf{P}^{2}} \mathbf{P}+\mathbf{K} \wedge \mathbf{W}\right)-\mathbf{J} \wedge(\mathbf{P} \wedge \mathbf{W})+\frac{3}{2} \mathbf{P} \wedge \mathbf{W}\right\} .
\end{aligned}
$$


Next we can deduce Poincaré operators as elements in $U(\mathcal{G})$. By using the relations (3.4)-(3.8) we find that

$$
c^{2} \mathbf{P}^{\prime 2}=H^{\prime 2} \quad c^{2} \mathbf{K}^{\prime} \wedge \mathbf{P}^{\prime}=H \mathbf{J}-H\left\{\frac{\mathbf{J} \cdot(\mathbf{P} \wedge \mathbf{W})}{\mathbf{P}^{2} \mathbf{W}^{2}}\right\} \mathbf{P} \wedge \mathbf{W} .
$$

Thus the Pauli-Lubanski components turn out to be

$$
W_{0}^{\prime}=\frac{1}{c} H \mathbf{J} \cdot \frac{(\mathbf{P} \wedge \mathbf{W})}{|\mathbf{P} \| \mathbf{W}|} \quad \mathbf{W}^{\prime}=-\frac{1}{c^{2}} H\left\{\frac{\mathbf{J} \cdot(\mathbf{P} \wedge \mathbf{W})}{|\mathbf{P} \| \mathbf{W}|}\right\} \frac{\mathbf{P} \wedge \mathbf{W}}{|\mathbf{P} \| \mathbf{W}|} .
$$

These expressions explicitly show how the component $W_{0}^{\prime}$, not appearing in the Galilei case (3.3), can be constructed and, moreover, they lead to

$$
\mathbf{W}^{\prime}=-\frac{1}{c} W_{0}^{\prime} \frac{\mathbf{P} \wedge \mathbf{W}}{|\mathbf{P} \| \mathbf{W}|} \quad c^{2} \mathbf{W}^{\prime 2}=W_{0}^{\prime 2} .
$$

If we now substitute these expresions into the Poincaré Casimirs $\mathcal{C}_{1}^{\prime}, \mathcal{C}_{2}^{\prime}$ given in (4.2) it can be checked that both of them vanish. This, in turn, means that under this deformation any irreducible Galilean representation with both $\mathcal{C}_{1} \neq 0, \mathcal{C}_{2} \neq 0$ gives rise to a massless and spinless Poincaré representation with both $\mathcal{C}_{1}^{\prime}=\mathcal{C}_{2}^{\prime}=0$. Hence, the expressions (4.7) can be interpreted as a correspondence between non-relativistic 'ordinary' systems and relativistic zero-mass systems. This suggests some kind of relationship between these operators and the null-plane (or light-cone) Poincaré framework [26].

Furthermore, a remarkable fact is the appearance in (4.7) of a Galilean angular momentum $[27]$

$$
\lambda_{p}=\frac{\mathbf{J} \cdot \mathbf{P}}{|\mathbf{P}|}=\mathbf{J} \cdot \mathbf{u}_{p}
$$

which generates an $\mathfrak{s o}(2)$ Lie algebra and corresponds to the projection of $\mathbf{J}$ along the direction $\mathbf{u}_{p}$ of the motion $\mathbf{P}$; recall that we have required $\mathcal{C}_{1}^{1 / 2}=|\mathbf{P}| \neq 0$. Similarly we can consider two other helicity-type operators defined along two orthogonal directions to $\mathbf{P}$ determined by $\mathbf{W}=\mathbf{K} \wedge \mathbf{P}$ and $\mathbf{P} \wedge \mathbf{W}$ (note also that accordingly $\mathcal{C}_{2}^{1 / 2}=|\mathbf{W}|$ has been assumed to be different from zero):

$$
\lambda_{w}:=\frac{\mathbf{J} \cdot \mathbf{W}}{|\mathbf{W}|}=\mathbf{J} \cdot \mathbf{u}_{w} \quad \lambda_{p w}:=\frac{\mathbf{J} \cdot(\mathbf{P} \wedge \mathbf{W})}{|\mathbf{P} \wedge \mathbf{W}|}=\mathbf{J} \cdot \mathbf{u}_{p w} .
$$

By taking into account (3.7) and (3.8), we find that these three Galilean 'angular momenta' close an $\mathfrak{s o}(3)$ Lie algebra:

$$
\left[\lambda_{w}, \lambda_{p}\right]=\lambda_{p w} \quad\left[\lambda_{p w}, \lambda_{w}\right]=\lambda_{p} \quad\left[\lambda_{p}, \lambda_{p w}\right]=\lambda_{w}
$$

In this way, we can write the Poincaré angular momentum $\lambda^{\prime}$ and the Pauli-Lubanski components in terms of the Galilean 'helicity' $\lambda_{p w}$ in the form

$$
\lambda^{\prime}=c \frac{W_{0}^{\prime}}{H^{\prime}}=\frac{\mathbf{J}^{\prime} \cdot \mathbf{P}^{\prime}}{\left|\mathbf{P}^{\prime}\right|} \equiv \lambda_{p w} \quad W_{0}^{\prime}=\frac{1}{c} H \lambda_{p w} \quad \mathbf{W}^{\prime}=-\frac{1}{c^{2}} H \lambda_{p w} \mathbf{u}_{p w} .
$$

Now we consider the Poincaré operators $Q_{i}^{\prime}$ defined by Bacry [28] (of course, for $H^{\prime} \neq$ $0)$ :

$$
Q_{i}^{\prime}=\frac{c^{2}}{2}\left(\frac{1}{H^{\prime}} K_{i}^{\prime}+K_{i}^{\prime} \frac{1}{H^{\prime}}\right)
$$


By introducing (4.7) and applying the relations (3.4)-(3.8) we find that these operators are expressed through elements of $U(\mathcal{G})$ as

$$
\begin{aligned}
\frac{1}{c} \mathbf{Q}^{\prime} & =\frac{|\mathbf{W}|}{\mathbf{P}^{2}} \frac{\mathbf{P}}{|\mathbf{P}|}+\frac{\mathbf{K} \wedge \mathbf{W}}{|\mathbf{P} \| \mathbf{W}|}+\frac{1}{H}\left(\frac{\mathbf{J} \cdot \mathbf{P}}{|\mathbf{P}|} \frac{\mathbf{W}}{|\mathbf{W}|}-\frac{\mathbf{J} \cdot \mathbf{W}}{|\mathbf{W}|} \frac{\mathbf{P}}{|\mathbf{P}|}+\frac{\mathbf{P} \wedge \mathbf{W}}{|\mathbf{P} \| \mathbf{W}|}\right) \\
& =\frac{|\mathbf{W}|}{\mathbf{P}^{2}} \mathbf{u}_{p}+\frac{\mathbf{K} \wedge \mathbf{W}}{|\mathbf{P} \| \mathbf{W}|}+\frac{1}{H}\left(\lambda_{p} \mathbf{u}_{w}-\lambda_{w} \mathbf{u}_{p}+\mathbf{u}_{p w}\right) .
\end{aligned}
$$

Some expected properties fulfilled by $\mathbf{Q}^{\prime}$ are consistently recovered under deformation. Working within $U(\mathcal{G})$ we obtain

$$
\left[J_{i}^{\prime}, Q_{j}^{\prime}\right]=\varepsilon_{i j k} Q_{k}^{\prime} \quad\left[Q_{i}^{\prime}, P_{j}^{\prime}\right]=\delta_{i j} .
$$

The former Lie bracket shows that $\mathbf{Q}^{\prime}$ behaves as a 3 -vector under rotations $\mathbf{J}^{\prime}=\mathbf{J}$, while the latter provides the natural canonical commutation relations between Galilean position and momenta operators; had we kept a definition for Galilean position operators $Q_{i}$ analogous to (4.15) then we would had obtained the unwanted result $\left[Q_{i}, P_{j}\right]=0$. Secondly, cumbersome computations lead to

$$
\begin{aligned}
{\left[Q_{i}^{\prime}, Q_{j}^{\prime}\right] } & =\frac{c^{2}}{H^{2}} \varepsilon_{i j k}\left(\frac{\mathbf{J} \cdot \mathbf{P}}{|\mathbf{P}|} \frac{P_{k}}{|\mathbf{P}|}+\frac{\mathbf{J} \cdot \mathbf{W}}{|\mathbf{W}|} \frac{W_{k}}{|\mathbf{W}|}-J_{k}\right) \\
& =\frac{c^{2}}{H^{\prime 2}}\left(Q_{i}^{\prime} P_{j}^{\prime}-Q_{j}^{\prime} P_{i}^{\prime}-\varepsilon_{i j k} J_{k}^{\prime}\right) .
\end{aligned}
$$

As a byproduct, the Poincaré kinematical observables $\Sigma_{k}^{\prime}=J_{k}^{\prime}-\varepsilon_{i j k} Q_{i}^{\prime} P_{j}^{\prime}$ can be written in terms of Galilean operators as

$$
\boldsymbol{\Sigma}^{\prime}=\mathbf{J}-\frac{\mathbf{J} \cdot \mathbf{P}}{|\mathbf{P}|} \frac{\mathbf{P}}{|\mathbf{P}|}-\frac{\mathbf{J} \cdot \mathbf{W}}{|\mathbf{W}|} \frac{\mathbf{W}}{|\mathbf{W}|}=\mathbf{J}-\lambda_{p} \mathbf{u}_{p}-\lambda_{w} \mathbf{u}_{w}
$$

Finally, the Poincaré velocity operator $\mathbf{V}^{\prime}$ turns out to be

$$
\mathbf{V}^{\prime}=\left[\mathbf{Q}^{\prime}, H^{\prime}\right]=c \frac{\mathbf{P} \wedge \mathbf{W}}{|\mathbf{P} \| \mathbf{W}|}=c^{2} \frac{\mathbf{P}^{\prime}}{H^{\prime}}=c \frac{\mathbf{P}^{\prime}}{\left|\mathbf{P}^{\prime}\right|}
$$

provided that $H^{\prime}=c\left|\mathbf{P}^{\prime}\right|$ since this deformation gives rise to Poincaré massless representations. The result (4.20) can be interpreted as a relationship between the motion of a non-relativistic particle along the direction $\mathbf{u}_{p w}$ with speed $c$ and the motion of a free massless relativistic particle along the direction $\mathbf{u}_{p^{\prime}}^{\prime}$.

\section{$5 \quad$ Newton-Hooke from centrally extended Galilei}

Besides Poincaré, the Galilei algebra has two other kinematical neighbours: the oscillating $\mathcal{N}_{+}$and the expanding $\mathcal{N}_{-}$NH algebras [16]. These are the Lie algebras of the motion groups of absolute time universes with non-zero curvature $\kappa_{1}= \pm 1 / \tau^{2}$ where $\tau$ is the universe time radius. The commutation rules of $\mathcal{N}_{ \pm}$are given by

$$
\begin{array}{lll}
{\left[J_{i}^{\prime}, J_{j}^{\prime}\right]=\varepsilon_{i j k} J_{k}^{\prime}} & {\left[J_{i}^{\prime}, P_{j}^{\prime}\right]=\varepsilon_{i j k} P_{k}^{\prime}} & {\left[J_{i}^{\prime}, K_{j}^{\prime}\right]=\varepsilon_{i j k} K_{k}^{\prime}} \\
{\left[P_{i}^{\prime}, P_{j}^{\prime}\right]=0} & {\left[P_{i}^{\prime}, K_{j}^{\prime}\right]=0} & {\left[K_{i}^{\prime}, K_{j}^{\prime}\right]=0} \\
{\left[H^{\prime}, P_{i}^{\prime}\right]=\kappa_{1} K_{i}^{\prime}} & {\left[H^{\prime}, K_{i}^{\prime}\right]=-P_{i}^{\prime}} & {\left[H^{\prime}, J_{i}^{\prime}\right]=0 .}
\end{array}
$$


The Casimirs of $\mathcal{N}_{ \pm}$turn out to be:

$$
\mathcal{C}_{1}^{\prime}=\mathbf{P}^{\prime 2}+\kappa_{1} \mathbf{K}^{\prime 2} \quad \mathcal{C}_{2}^{\prime}=\mathbf{W}^{\prime 2}
$$

where the components of $\mathbf{W}^{\prime}$ are formally identical to the Galilean ones (3.3).

Due to the non-zero Lie brackets $\left[H^{\prime}, P_{i}^{\prime}\right]=\kappa_{1} K_{i}^{\prime}$, the vector subspaces $\mathfrak{p}^{(l)}$ of the Cartan decompositions (3.10) now verify $\left[\mathfrak{p}^{(1)}, \mathfrak{p}^{(1)}\right] \subset \mathfrak{h}^{(1)}$ and $\left[\mathfrak{p}^{(2)}, \mathfrak{p}^{(2)}\right]=0$. The NH groups $N_{ \pm}$are the motion groups of the following symmetric homogeneous spaces

$$
\begin{array}{ll}
\mathcal{S}^{(1)}=N_{+} / H^{(1)}=T_{6}(S O(2) \otimes S O(3)) / I S O(3) & \kappa_{1}=1 / \tau^{2} \\
\mathcal{S}^{(2)}=N_{+} / H^{(2)}=T_{6}(S O(2) \otimes S O(3)) /(S O(2) \otimes S O(3)) & \kappa_{2}=0 \\
\mathcal{S}^{(1)}=N_{-} / H^{(1)}=T_{6}(S O(1,1) \otimes S O(3)) / I S O(3) & \kappa_{1}=-1 / \tau^{2} \\
\mathcal{S}^{(2)}=N_{-} / H^{(2)}=T_{6}(S O(1,1) \otimes S O(3)) /(S O(1,1) \otimes S O(3)) & \kappa_{2}=0 .
\end{array}
$$

The spaces $\mathcal{S}^{(1)}$ and $\mathcal{S}^{(2)}$ correspond, in this order, to the $(3+1) \mathrm{D}$ non-relativistic curved spacetime $[24,29]$ and the $6 \mathrm{D}$ flat phase space (or space of time-like worldlines in the spacetime).

The limit $\kappa_{1} \rightarrow 0(\tau \rightarrow \infty)$ produces the spacetime contraction $\mathcal{N}_{ \pm} \rightarrow \mathcal{G}$. We consider the opposite process which would introduce a universe time radius $\tau$ in $U(\mathcal{G})$. As we are dealing with rank-one deformations we only consider the first Casimir (5.2), so that the 'seed' element (2.2) is simply $\mathcal{J}=\alpha_{1} \mathcal{J}_{1}^{(1)}=\alpha_{1} \mathbf{K}^{2}$. The deformed generators (2.3) are $H^{\prime}=2 \alpha_{1} \mathbf{K} \cdot \mathbf{P}$ and all the remaining ones unchanged. However these new generators $d o$ not $\operatorname{span} \mathcal{N}_{ \pm}$. Hence it is necessary to take an initial Lie algebra less abelian in order to be able to perform the deformation. Thus we start from a central extension of $\mathcal{G}$ (as in lower dimensions $[14,30]$ ), with central generator $\Xi$ and central charge $m$ (the mass of the Galilean particle as a Galilean elementary system [15]). The commutation rules of the centrally extended Galilei algebra $\overline{\mathcal{G}}$ are given by (3.1) once the vanishing brackets $\left[K_{i}, P_{j}\right]$ are replaced by

$$
\left[K_{i}, P_{j}\right]=\delta_{i j} m \Xi \quad[\Xi, \cdot]=0 .
$$

We now apply (2.3) with $\mathcal{J}=\alpha_{1} \mathbf{K}^{2}$ for the Lie brackets of $\overline{\mathcal{G}}$, finding the deformed generators, which live on the universal enveloping algebra $U(\overline{\mathcal{G}})$ :

$$
J_{i}^{\prime}=J_{i} \quad K_{i}^{\prime}=K_{i} \quad H^{\prime}=2 \alpha_{1} \mathbf{K} \cdot \mathbf{P}-3 \alpha_{1} m \Xi \quad P_{i}^{\prime}=2 \alpha_{1} m \Xi K_{i} .
$$

Then this deformation is characterized by:

Proposition 2. The generators defined in terms of the (extended, with $m \neq 0$ ) Galilean ones by (5.4) span the $(3+1) D N H$ algebras provided that the constant $\alpha_{1}$ fulfils

$$
\alpha_{1}^{2}=-\frac{\kappa_{1}}{4 m^{2} \Xi^{2}}=\mp \frac{1}{4 \tau^{2} m^{2} \Xi^{2}} \quad \text { for } \quad \mathcal{N}_{ \pm} .
$$

Proof: The generators which are unchanged in the deformation close the isotopy subalgebra of an event $\mathfrak{h}^{(1)}=\langle\mathbf{K}, \mathbf{J}\rangle$. Thus the proposition 1 of [14] can be applied and we only need to compute the Lie brackets involving generators of $\mathfrak{p}^{(1)}$. By direct computations we obtain the relation (5.5):

$$
\left[P_{i}^{\prime}, P_{j}^{\prime}\right]=0 \quad\left[H^{\prime}, P_{i}^{\prime}\right]=-4 \alpha_{1}^{2} m^{2} \Xi^{2} K_{i} \equiv \kappa_{1} K_{i}^{\prime} .
$$

Therefore this deformation introduces a constant curvature $\kappa_{1}$ in the flat spacetime $G / H^{(1)}$, leading to curved spacetimes $N_{ \pm} / H^{(1)}$ with the transition $\left[\mathfrak{p}^{(1)}, \mathfrak{p}^{(1)}\right]=0 \rightarrow$ $\left[\mathfrak{p}^{(1)}, \mathfrak{p}^{(1)}\right] \subset \mathfrak{h}^{(1)}$. All the phase spaces $\mathcal{S}^{(2)}$ remain flat. 


\subsection{Deformation of Galilei representations}

If we set $\alpha_{1}=\sqrt{-\kappa_{1}} /(2 m \Xi)$ and substitute in the deformed generators (5.4), we find that these read

$$
H^{\prime}=\sqrt{-\kappa_{1}}\left(\frac{\mathbf{K} \cdot \mathbf{P}}{m \Xi}-\frac{3}{2}\right) \quad \mathbf{P}^{\prime}=\sqrt{-\kappa_{1}} \mathbf{K} .
$$

Hence $\mathcal{C}_{1}^{\prime}=0$ and $\mathbf{W}^{\prime}=\mathbf{K}^{\prime} \wedge \mathbf{P}^{\prime}=0$. Consequently, any irreducible representation of $\overline{\mathcal{G}}$ with $m \neq 0$ gives rise to a representation of $\mathcal{N}_{ \pm}$with zero Casimirs eigenvalues as both deformed $\mathcal{C}_{l}^{\prime}$ (5.2) vanish. In particular, let us consider the representation of $\overline{\mathcal{G}}$ in terms of the momenta $\mathbf{p}=\left(p_{1}, p_{2}, p_{3}\right)$ given by Bacry [27]:

$$
J_{i}=\varepsilon_{i j k} p_{k} \frac{\partial}{\partial p_{j}}+S_{i} \quad K_{i}=m \frac{\partial}{\partial p_{i}} \quad H=\frac{\mathbf{p}^{2}}{2 m}+a, \quad P_{i}=p_{i} \quad \Xi=1
$$

where $a$ is a constant and $S_{i}$ are spin operators fulfilling $\left[S_{i}, S_{j}\right]=\varepsilon_{i j k} S_{k}$. Then the relations (5.6) lead to the following representation for the transformed generators of $\mathcal{N}_{ \pm}$:

$$
H^{\prime}=\sqrt{-\kappa_{1}}\left(\mathbf{p} \cdot \frac{\partial}{\partial \mathbf{p}}+\frac{3}{2}\right) \quad \mathbf{P}^{\prime}=\sqrt{-\kappa_{1}} m \frac{\partial}{\partial \mathbf{p}}
$$

keeping $\mathbf{J}^{\prime}=\mathbf{J}$ and $\mathbf{K}^{\prime}=\mathbf{K}$.

\section{6 (Anti)de Sitter from Poincaré}

Known Lie algebra deformations $i \mathfrak{s o}(p, q) \rightarrow \mathfrak{s o}(p+1, q)[7,9]$ can be recovered from our approach, which also provides the explicit form of the operators representing the deformed generators inside the initial universal enveloping algebra. As an example, in this section we give either anti-de Sitter $\mathcal{S}_{+}=\mathfrak{s o}(3,2)$ or de Sitter $\mathcal{S}_{-}=\mathfrak{s o}(4,1)$ algebras and representations as deformations of Poincaré ones. To maintain consistency with the notation in the previous part of the paper, we will denote here $X^{\prime}$ any element of $U(\mathcal{P})$ (as (4.1) and (4.2)) and $X^{\prime \prime}$ any (anti)de Sitter object. The (anti)de Sitter commutation relations read

$$
\begin{array}{lll}
{\left[J_{i}^{\prime \prime}, J_{j}^{\prime \prime}\right]=\varepsilon_{i j k} J_{k}^{\prime \prime}} & {\left[J_{i}^{\prime \prime}, P_{j}^{\prime \prime}\right]=\varepsilon_{i j k} P_{k}^{\prime \prime}} & {\left[J_{i}^{\prime \prime}, K_{j}^{\prime \prime}\right]=\varepsilon_{i j k} K_{k}^{\prime \prime}} \\
{\left[P_{i}^{\prime \prime}, P_{j}^{\prime \prime}\right]=-\frac{\kappa_{1}}{c^{2}} \varepsilon_{i j k} J_{k}^{\prime \prime}} & {\left[P_{i}^{\prime \prime}, K_{j}^{\prime \prime}\right]=-\frac{1}{c^{2}} \delta_{i j} H^{\prime \prime}} & {\left[K_{i}^{\prime \prime}, K_{j}^{\prime \prime}\right]=-\frac{1}{c^{2}} \varepsilon_{i j k} J_{k}^{\prime \prime}} \\
{\left[H^{\prime \prime}, P_{i}^{\prime \prime}\right]=\kappa_{1} K_{i}^{\prime \prime}} & {\left[H^{\prime \prime}, K_{i}^{\prime \prime}\right]=-P_{i}^{\prime \prime}} & {\left[H^{\prime \prime}, J_{i}^{\prime \prime}\right]=0}
\end{array}
$$

where $\kappa_{1}$ is the curvature of the spacetime: $\kappa_{1}=1 / \tau^{2}$ for $\mathcal{S}_{+}$and $\kappa_{1}=-1 / \tau^{2}$ for $\mathcal{S}_{-}$. In both cases the constant $\tau$ plays the role of the curvature radius of the (anti)de Sitter universe; we recall that in our conventions $\tau$ is dimensionally a time (so the "length radius' would be $c \tau$ ) and the sign of spacetime curvature has been chosen so that $\kappa_{1}$ is related to the acceleration of separation of time-like geodesics with the same sign as in the Riemannian case (the sign is opposite for space-like geodesics). The Casimir invariants are [22]:

$$
\begin{aligned}
& \mathcal{C}_{1}^{\prime \prime}=\mathbf{P}^{\prime \prime 2}-\frac{1}{c^{2}} H^{\prime \prime 2}+\kappa_{1}\left(\mathbf{K}^{\prime \prime 2}-\frac{1}{c^{2}} \mathbf{J}^{\prime \prime 2}\right) \\
& \mathcal{C}_{2}^{\prime \prime}=\mathbf{W}^{\prime \prime 2}-\frac{1}{c^{2}} W_{0}^{\prime \prime 2}-\frac{\kappa_{1}}{c^{2}}\left(\mathbf{J}^{\prime \prime} \cdot \mathbf{K}^{\prime \prime}\right)^{2}
\end{aligned}
$$


where $\mathbf{W}^{\prime \prime}, W_{0}^{\prime \prime}$ are formally identical to the Poincaré Pauli-Lubanski components (4.2). Now, both vector subspaces $\mathfrak{p}^{(l)}(3.10)$ fulfil $\left[\mathfrak{p}^{(l)}, \mathfrak{p}^{(l)}\right] \subset \mathfrak{h}^{(l)}$, and the symmetric homogeneous (anti)de Sitter spaces are defined by

$$
\begin{array}{ll}
\mathcal{S}^{(1)}=A d S / H^{(1)}=S O(3,2) / S O(3,1) & \kappa_{1}=1 / \tau^{2} \\
\mathcal{S}^{(2)}=A d S / H^{(2)}=S O(3,2) /(S O(2) \otimes S O(3)) & \kappa_{2}=-1 / c^{2} \\
\mathcal{S}^{(1)}=d S / H^{(1)}=S O(4,1) / S O(3,1) & \kappa_{1}=-1 / \tau^{2} \\
\mathcal{S}^{(2)}=d S / H^{(2)}=S O(4,1) /(S O(1,1) \otimes S O(3)) & \kappa_{2}=-1 / c^{2} .
\end{array}
$$

Hence the spaces $\mathcal{S}^{(1)}$ are the curved relativistic spacetimes (with the two possible signs of the curvature), and the two 'phase spaces' $\mathcal{S}^{(2)}$ are the corresponding spaces of time-like lines; both are (negatively) curved phase spaces.

The deformation $U(\mathcal{P}) \rightarrow\left\{\mathcal{S}_{ \pm}\right\}$is rank-one with underlying space $\mathcal{S}^{(1)}$ and curvature $\kappa_{1}$. Then only the first Casimir $\mathcal{C}_{1}^{\prime \prime}$ (6.2) determines the 'seed' element (2.2):

$$
\mathcal{J}=\alpha_{1}\left(\mathbf{K}^{\prime \prime 2}-\frac{1}{c^{2}} \mathbf{J}^{\prime \prime 2}\right)
$$

so that the deformed generators, defined on $U(\mathcal{P})$, turn out to be

$$
\begin{aligned}
& J_{i}^{\prime \prime}=J_{i}^{\prime} \quad K_{i}^{\prime \prime}=K_{i}^{\prime} \quad H^{\prime \prime}=\frac{2 \alpha_{1}}{c^{2}}\left(c^{2} \mathbf{K}^{\prime} \cdot \mathbf{P}^{\prime}-\frac{3}{2} H^{\prime}\right) \\
& P_{i}^{\prime \prime}=\frac{2 \alpha_{1}}{c^{2}}\left(K_{i}^{\prime} H^{\prime}+\varepsilon_{i j k} J_{j}^{\prime} P_{k}^{\prime}-\frac{3}{2} P_{i}^{\prime}\right) .
\end{aligned}
$$

The constant $\alpha_{1}$ is determined similarly to the NH case.

Proposition 3. For a given irreducible representation of the Poincaré algebra with secondorder Poincaré Casimir $\mathcal{C}_{1}^{\prime} \neq 0$, the generators defined by (6.4) close the $(3+1) D$ (anti)de Sitter algebras whenever the constant $\alpha_{1}$ is related to the value $\mathcal{C}_{1}^{\prime}$ by

$$
\alpha_{1}^{2}=\frac{\kappa_{1} c^{2}}{4 \mathcal{C}_{1}^{\prime}}= \pm \frac{c^{2}}{4 \tau^{2} \mathcal{C}_{1}^{\prime}}
$$

Notice that within our approach light-like representations of the Poincaré algebra cannot provide deformations to (anti)de Sitter representations.

Proof: As in the previous section the isotopy subalgebra $\mathfrak{h}^{(1)}$ of an event, remains invariant under this deformation, thus we only compute the Lie brackets for the translation generators that belong to $\mathfrak{p}^{(1)}$; these directly give (6.5):

$$
\left[P_{i}^{\prime \prime}, P_{j}^{\prime \prime}\right]=-\frac{4 \alpha_{1}^{2}}{c^{4}} \varepsilon_{i j k} \mathcal{C}_{1}^{\prime} J_{k}^{\prime} \equiv-\frac{\kappa_{1}}{c^{2}} \varepsilon_{i j k} J_{k}^{\prime \prime} \quad\left[H^{\prime \prime}, P_{i}^{\prime \prime}\right]=\frac{4 \alpha_{1}^{2}}{c^{2}} \mathcal{C}_{1}^{\prime} K_{i}^{\prime} \equiv \kappa_{1} K_{i}^{\prime \prime}
$$

\subsection{Deformation of Poincaré representations}

Further to the above algebraic results, which are in agreement with those established in $[7,9]$, we explore the deformation of representations. 
By taking into account the deformed generators (6.4) with the solution (6.5), it can be proven that the (anti)de Sitter invariants (6.2) are expressed in terms of the Poincaré Casimirs (4.2) as

$$
\mathcal{C}_{1}^{\prime \prime}=-\frac{9 \kappa_{1}}{4 c^{2}}+\kappa_{1} \frac{\mathcal{C}_{2}^{\prime}}{\mathcal{C}_{1}^{\prime}} \quad \mathcal{C}_{2}^{\prime \prime}=-\frac{\kappa_{1}}{4 c^{2}} \frac{\mathcal{C}_{2}^{\prime}}{\mathcal{C}_{1}^{\prime}}
$$

Since this Poincaré deformation is only possible when $\mathcal{C}_{1}^{\prime} \neq 0$, the result (6.6) means that, in general, any massive Poincaré particle gives rise generically to an (anti)de Sitter representation with $\mathcal{C}_{1}^{\prime \prime} \neq 0$, with a singular behaviour when $\mathcal{C}_{2}^{\prime}=9 \mathcal{C}_{1}^{\prime} /\left(4 c^{2}\right)$ which gives $\mathcal{C}_{1}^{\prime \prime}=0$. As far as the second Casimir it is clear that $\mathcal{C}_{2}^{\prime}=0$ leads to $\mathcal{C}_{2}^{\prime \prime}=0$.

In particular, let us consider the irreducible Poincaré representation of mass $m \neq 0$ and spin $s \neq 0$ in the momentum $\mathbf{p}$-space [27]:

$$
\begin{aligned}
& H^{\prime}=c \sqrt{\mathbf{p}^{2}+m^{2} c^{2}} \quad P_{i}^{\prime}=p_{i} \quad J_{i}^{\prime}=\varepsilon_{i j k} p_{k} \frac{\partial}{\partial p_{j}}+S_{i} \\
& K_{i}^{\prime}=\frac{1}{c}\left(\sqrt{\mathbf{p}^{2}+m^{2} c^{2}} \frac{\partial}{\partial p_{i}}+\frac{\varepsilon_{i j k} p_{j} S_{k}}{m c+\sqrt{\mathbf{p}^{2}+m^{2} c^{2}}}\right)
\end{aligned}
$$

where $S_{i}$ are spin operators such that the eigenvalue of $\mathbf{S}^{2}$ is $s(s+1)$; note that $p^{0}=$ $c \sqrt{\mathbf{p}^{2}+m^{2} c^{2}}$. This representation is characterized by the eigenvalues of both Casimirs $\mathcal{C}_{l}^{\prime}$ $(4.2)$ :

$$
\mathcal{C}_{1}^{\prime}=-m^{2} c^{2} \quad \mathcal{C}_{2}^{\prime}=m^{2} \mathbf{S}^{2}
$$

Then we find that the eigenvalues of the (anti)de Sitter Casimirs (6.6) read

$$
\mathcal{C}_{1}^{\prime \prime}=-\frac{\kappa_{1}}{c^{2}}\left(\frac{9}{4}+\mathbf{S}^{2}\right) \quad \mathcal{C}_{2}^{\prime \prime}=\frac{\kappa_{1}}{4 c^{4}} \mathbf{S}^{2}
$$

so that, in this case $\mathcal{C}_{1}^{\prime \prime} \neq 0$ for any non-vanishing spin. Finally, by taking $\alpha_{1}=\sqrt{-\kappa_{1}} /(2 m)$, the deformed generators (6.4) yield to the following (anti)de Sitter representation

$$
\begin{aligned}
& H^{\prime \prime}=\frac{\sqrt{-\kappa_{1}}}{m c} \sqrt{\mathbf{p}^{2}+m^{2} c^{2}}\left(\mathbf{p} \cdot \frac{\partial}{\partial \mathbf{p}}+\frac{3}{2}\right) \\
& P_{i}^{\prime \prime}=\sqrt{-\kappa_{1}}\left(\frac{p_{i}}{m c^{2}}\left(\mathbf{p} \cdot \frac{\partial}{\partial \mathbf{p}}+\frac{3}{2}\right)+m \frac{\partial}{\partial p_{i}}-\frac{\varepsilon_{i j k} p_{j} S_{k}}{m c^{2}+c \sqrt{\mathbf{p}^{2}+m^{2} c^{2}}}\right)
\end{aligned}
$$

with $\mathbf{J}^{\prime \prime}=\mathbf{J}^{\prime}$ and $\mathbf{K}^{\prime \prime}=\mathbf{K}^{\prime}$.

\section{Outlook: Beyond classical deformations}

The deformation method proposed in [14] for the $(2+1) \mathrm{D}$ kinematical algebras has been generalized in order to be applicable to higher dimensions and to any Lie algebra, and has been explicitly applied to the $(3+1) \mathrm{D}$ Galilei algebra. The method works in the two physically meaningful 'deformation directions' which lead either to the Poincaré algebra or to the NH ones. Deformations going from Poincaré to both de Sitter algebras have been recovered as well. Furthermore, beyond the pure algebraic constructions, we have presented a preliminary study of deformations of operators and representations which may deserve deeper development. 
For the deformation from Galilei to Poincaré, the method as described here does not provide all the Poincaré representations, but only the 'light-like' ones associated to the vanishing of both Poincaré Casimirs. To extend the method so as to allow the general representations of the Poincaré algebra remains as an open problem.

Clearly, the same procedure also holds for the rank-two deformation going from Galilei to the Euclidean algebra $\mathcal{E} \equiv \mathfrak{i s o}(4)$. If we replace in all the Poincaré expressions given in section 4 , the negative curvature $\kappa_{2}=-1 / c^{2}$ by a positive one (set $c$ equal to a pure imaginary complex number), then we would obtain all the Euclidean structures. The deformation $U(\mathcal{G}) \rightarrow \mathcal{E}$ would lead to similar deformed generators and conditions as those characterized by the proposition 1 . In this case, the symmetric space $\mathcal{S}^{(1)}=I S O(4) / S O(4)$ is the $4 \mathrm{D}$ flat Euclidean space and $\mathcal{S}^{(2)}=I S O(4) /(\mathbb{R} \otimes S O(3))$ is a positively curved and rank-two 6D space of lines in the Euclidean space. Deformation of Galilean representations are characterized by the deformed Euclidean Casimirs $\mathcal{C}_{l}^{\prime}$ whose eigenvalues are again equal to zero. Summing up, we have obtained the following rank-two Galilei deformations:

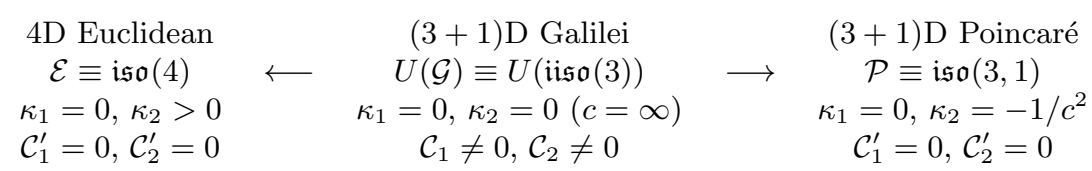

In the same way, similar rank-one deformations to those described in section 6 but now starting from $U(\mathcal{E})$ instead of $U(\mathcal{P})$ can also be obtained. They would give rise to either $\mathfrak{s o}(5)\left(\kappa_{1}>0\right)$ or $\mathfrak{s o}(4,1)\left(\kappa_{1}<0\right)$, with underlying homogeneous spaces $\mathcal{S}^{(l)}$ given in this order by: (i) the $4 \mathrm{D}$ sphere $\mathcal{S}^{(1)}=S O(5) / S O(4)$ with radius $R$ and curvature $\kappa_{1}=1 / R^{2}$, and the Grassmannian $\mathcal{S}^{(2)}=S O(5) /(S O(2) \otimes S O(3))$ with $\kappa_{2}>0$; (ii) the $4 \mathrm{D}$ hyperbolic space $\mathcal{S}^{(1)}=S O(4,1) / S O(4)$, with $\kappa_{1}=-1 / R^{2}$ and the corresponding grassmannian $\mathcal{S}^{(2)}=S O(4,1) /(S O(1,1) \otimes S O(3))$ with $\kappa_{2}>0$. In both processes the eigenvalue of the Euclidean Casimir $\mathcal{C}_{1}^{\prime}$ must be different from zero and deformation of Euclidean representations are determined by the deformed Casimirs $\mathcal{C}_{l}^{\prime \prime}(6.6)$ with $c$ purely imaginary (say $c=\mathrm{i}$, so that $\kappa_{2}=+1$ ). Hence the rank-one deformations studied here (covering sections 5 and 6 ) are summarized as:

$$
\begin{aligned}
& \text { 4D Sphere 4D Euclidean }
\end{aligned}
$$

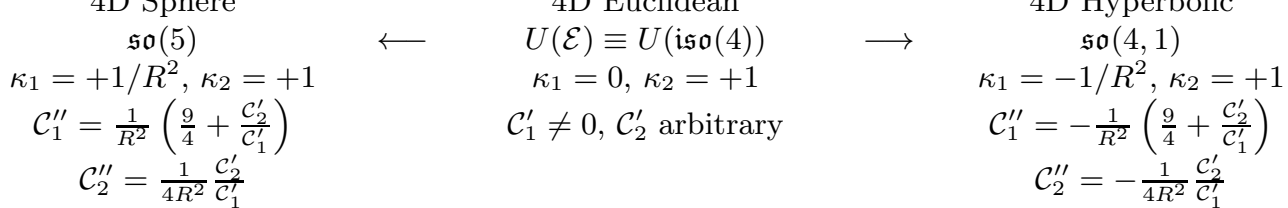

$$
\begin{aligned}
& (3+1) \mathrm{D} \text { Anti-de Sitter } \quad(3+1) \mathrm{D} \text { Poincaré } \quad(3+1) \mathrm{D} \text { de Sitter }
\end{aligned}
$$

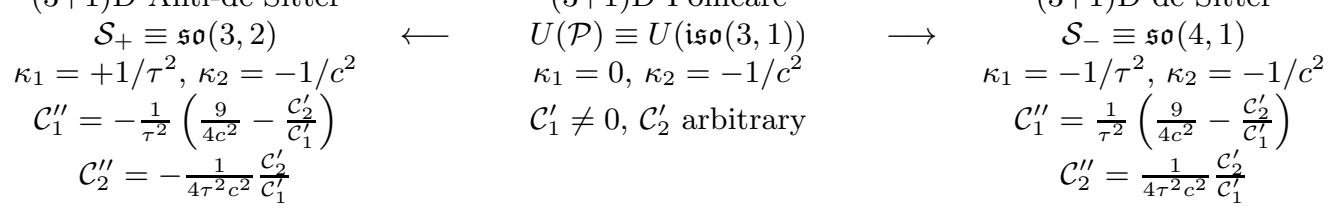

$$
\begin{aligned}
& (3+1) \text { D Oscillating } \mathrm{NH} \\
& \mathcal{N}_{+} \equiv \mathfrak{t}_{6}(\mathfrak{s o}(2) \oplus \mathfrak{s o}(3)) \\
& \kappa_{1}=+1 / \tau^{2}, \kappa_{2}=0 \\
& \mathcal{C}_{1}^{\prime}=0, \mathcal{C}_{2}^{\prime}=0 \\
& \begin{array}{c}
(3+1) \mathrm{D} \text { Extended Galilei } \\
U(\overline{\mathcal{G}}) \equiv U(\overline{\mathfrak{i i s o}(3)}) \\
\kappa_{1}=0, \kappa_{2}=0 \\
m \neq 0
\end{array} \\
& (3+1) \text { D Expanding } \mathrm{NH} \\
& \longrightarrow \quad \mathcal{N}_{-} \equiv \mathfrak{t}_{6}(\mathfrak{s o}(1,1) \oplus \mathfrak{s o}(3)) \\
& \kappa_{1}=-1 / \tau^{2}, \kappa_{2}=0 \\
& \mathcal{C}_{1}^{\prime}=0, \mathcal{C}_{2}^{\prime}=0
\end{aligned}
$$

It is worth remarking that each of the above deformations introduces a new parameter 
$\kappa$ in the initial Lie algebra and associated structures which, from the very beginning of our construction, plays the role of a constant curvature of some underlying symmetrical homogeneous space. In particular, in the deformation from the Galilean spacetime to the relativistic one, this process can alternatively be seen as the introduction of a fundamental scale $c\left(\kappa_{2}=-1 / c^{2}\right)$. The sequence of these classical deformations has a natural end when the semisimple Lie algebras $\mathfrak{s o}(p, q)$ are reached. In this respect, we would like to stress that the aforementioned geometrical and physical ideas behind these constructions can also be found in the framework of quantum algebras or $q$-deformations [31] $U_{z}(\mathfrak{g})$ of a Lie algebra $\mathfrak{g}$. These also make use of its universal enveloping algebra $U(\mathfrak{g})$ together with a deformation parameter $z\left(q=\mathrm{e}^{z}\right)$ and the classical limit $z \rightarrow 0$ leads to $U(\mathfrak{g})$. By one hand, quantum deformations of the Poincaré algebra have been used to obtain the so called 'doubly special relativities' (see [32, 33] and references therein) for which the deformation parameter $z$ is interpreted as a second fundamental scale related to the Planck length; thus this process is 'similar' to the introduction of $c$ through a Galilean deformation but obviously goes beyond Lie structures. On the other hand, quantum algebras have recently been shown to provide spaces of generically variable curvature which is governed by $z$ [34]. Hence when classical and quantum deformations are considered in a global picture one

finds a natural 'common' deformation setting which indicates the relevance of the use of universal enveloping algebras.

\section{Acknowledgments}

Comments and suggestions from a referee and from the adjudicator have been very helpful and are gratefully acknowledged. This work was partially supported by the Ministerio de Educación y Ciencia (Spain, Projects FIS2004-07913 and MTM2005-09183) and by the Junta de Castilla y León (Spain, Project VA013C05).

\section{References}

[1] Inönü E and Wigner E P 1953 Proc. Natl. Acad. Sci. USA 39510 Inönü E and Wigner E P 1954 Proc. Natl. Acad. Sci. USA 40119

[2] Segal I E 1951 Duke Math. J. 18221

[3] Saletan E J 1961 J. Math. Phys. 21

[4] Moody R V and Patera J 1991 J. Phys. A: Math. Gen. 242227

[5] de Montigny M and Patera J 1991 J. Phys. A: Math. Gen. 24525

[6] Weimar-Woods E 1991 J. Math. Phys. 322028 Weimar-Woods E 1995 J. Math. Phys. 364519

[7] Gilmore R 1974 Lie Groups, Lie Algebras and Some of Their Applications (New York: Wiley)

[8] Gerstenhaber M 1964 Ann. Math. 7959

Gerstenhaber M 1966 Ann. Math. 841 
Gerstenhaber M 1968 Ann. Math. 881

Gerstenhaber M 1974 Ann. Math. 99257

[9] Rosen J and Roman P 1966 J. Math. Phys. 72072

[10] Levy-Nahas M 1967 J. Math. Phys. 81211

[11] Nijenhuis A and Richardson R W 1967 J. Math. Mech. 1789

[12] Bayen F, Flato M, Fronsdal C, Lichnerowicz A and Sternheimer D 1977 Lett. Math. Phys. 1521

Bayen F, Flato M, Fronsdal C, Lichnerowicz A and Sternheimer D 1978 Ann. Phys. 11161

Bayen F, Flato M, Fronsdal C, Lichnerowicz A and Sternheimer D 1978 Ann. Phys. 111111

[13] Wolf K B and Boyer C P 1974 J. Math. Phys. 152096

[14] Herranz F J and Santander M 1999 J. Phys. A: Math. Gen. 323743

[15] Lévy-Leblond J M 1963 J. Math. Phys. 4776

Lévy-Leblond J M 1971 Galilei group and Galilean invariance in Group Theory and its applications ed E M Loebl (New York: Academic)

[16] Bacry H and Lévy-Leblond J M 1968 J. Math. Phys. 91605

[17] Bacry H and Nuyts J 1986 J. Math. Phys. 272455

[18] de Montigny M, Patera J and Tolar J 1994 J. Math. Phys. 35405

[19] Herranz F J and Santander M 1996 J. Phys. A: Math. Gen. 296643

[20] Figueroa-O'Farrill J M 1989 J. Math. Phys. 302735

[21] Fialowski A and de Montigny M 2005 J. Phys. A: Math. Gen. 386335

[22] Herranz F J and Santander M 1997 J. Phys. A: Math. Gen. 305411

[23] Jordan C 1961-1964 Essai sur la géométrie à $n$ dimensions, Oeuvres, (París: Gauthier-Villars)

[24] Herranz F J and Santander M 1998 Geometría y Física vol. XXXII ed J F Cariñena, E Martínez and M F Rañada (Madrid: Real Academia de Ciencias) p 59 Preprint physics $/ 9702030$

[25] Kirillov A A 2004 Lectures on the orbit method Grad. Studies in Math. vol. 64 (Providence RI: Am. Math. Soc.)

[26] Leutwyler H and Stern J 1978 Ann. Phys. (N.Y.) 112392

[27] Bacry H 1967 Leçons sur la théorie des groupes et les symétries des particules élémentaires (Paris: Dunod) (English translation, London: Gordon and Breach)

[28] Bacry H 1988 Ann. Inst. H. Poincaré 49245

Bacry H 1993 J. Phys. A: Math. Gen. 265413 
[29] Aldrovandi R, Barbosa A L, Crispino L C B and Pereida J G 1999 Classical Quant. Grav. 16495

[30] Nieto L M, Negro J and Santander M 1997 Int. J. Mod. Phys. A 12259

[31] Chari V and Pressley A 1994 A Guide to Quantum Groups (Cambridge: Cambridge University Press)

[32] Lukierski J and Nowicki A 2003 Int. J. Mod. Phys. A 187

[33] Ahluwalia-Khalilova D V 2005 Class. Quantum Grav. 221433

[34] Ballesteros A, Herranz F J and Ragnisco O 2005 Phys. Lett. B 610107

Ballesteros A, Herranz F J and Ragnisco O 2005 J. Phys. A: Math. Gen. 387129 